\title{
MicroRNA-146a expression inhibits the proliferation and promotes the apoptosis of bronchial smooth muscle cells in asthma by directly targeting the epidermal growth factor receptor
}

\author{
YANXIA ZHANG ${ }^{1}$, YAN XUE ${ }^{2}$, YAN LIU ${ }^{3}$, GUODONG SONG $^{4}$, GUOFENG LV $^{1}$, \\ YONGQIANG WANG ${ }^{1}$, YIJIANG WANG ${ }^{1},{\text { XIANG } \text { LI }^{1}}^{1}$ and LEIYING YANG ${ }^{5}$ \\ ${ }^{1}$ Department of Pediatrics, Maternal and Child Healthcare Hospital, Laiwu, Shandong 271100; \\ ${ }^{2}$ Department of Pediatrics, General Hospital of Yanzhou Mining Bureau, Jining, Shandong 272100; \\ ${ }^{3}$ Department of Pediatrics, Zoucheng People's Hospital, Zoucheng, Shandong 273500; \\ ${ }^{4}$ Department of Pediatrics, Dezhou People's Hospital, Dezhou, Shandong 253000; \\ ${ }^{5}$ Department of Pathology, Taishan Medical College, Taian, Shandong 271016, P.R. China
}

Received March 13, 2015; Accepted April 19, 2016

DOI: $10.3892 / \mathrm{etm} .2016 .3427$

\begin{abstract}
The present study aimed to determine the expression of microRNA-146a (miR-146a) in the plasma of children with asthma, and to investigate the effect of miR-146a on the proliferation and apoptosis of bronchial smooth muscle cells (BSMCs). Reverse transcription-quantitative polymerase chain reaction was used to determine the expression levels of miR-146a mimics and its inhibitor. A Cell Counting kit- 8 assay was performed to examine the proliferation of BSMCs. Caspase-3/7 activity was determined using a Caspase-Glo 3/7 kit. To measure the expression levels of proteins associated with apoptosis, western blotting was performed. The target gene of miR-146a was identified using a dual-luciferase reporter assay. The plasma levels of miR-146a in children with asthma were significantly higher compared with those in healthy children. Enhanced miR-146a expression inhibited the proliferation of BSMCs. BSMC apoptosis was promoted by miR-146a. The mechanism underlying the miR-146a-induced promotion of BSMC apoptosis may be its direct targeting of epidermal growth factor receptor (EGFR), which affects downstream signaling pathways. In conclusion, miR-146a expression in asthma inhibits the proliferation and promotes the apoptosis of BSMCs by direct targeting of EGFR.
\end{abstract}

Correspondence to: Professor Leiying Yang, Department of Pathology, Taishan Medical College, 619 Changcheng Road, Taian, Shandong 271016, P.R. China

E-mail: yly777@yeah.net

Key words: microRNA-146a, bronchial smooth muscle cell, proliferation, apoptosis

\section{Introduction}

Pediatric asthma is a common chronic respiratory system disease in children, with its main characteristics being inflammation, bronchial hyperreactivity and airway remodeling (1). Airway remodeling is an important step in the occurrence of asthma, and is associated with the severity of the disease (2). The abnormal proliferation of bronchial smooth muscle cells (BSMCs) has important roles in airway remodeling $(2,3)$. Previous studies reported that BSMCs in patients with bronchial asthma secrete more cytokines than those in normal subjects, including chemokine (C-X-C motif) ligand 10 and chemokine (C-X3-C motif) ligand $1(4,5)$. These cytokines induce the attachment of mast cells to BSMCs, promote the survival and proliferation of mast cells, facilitate the accumulation of inflammatory substances, thicken smooth muscles and lead to airway hyperreactivity. Previous investigations also demonstrated that attachment of T lymphocytes to BSMCs induces DNA synthesis in the BSMCs, which enhances their proliferation $(6,7)$. Enhanced proliferation and decreased apoptosis of BSMCs may result in airway hyperreactivity $(6,8)$.

MicroRNA (miRNA or miR) is a type of 18-22 nt non-encoding RNA that participates in the regulation of cell proliferation, apoptosis and differentiation (9). Numerous miRNAs are involved in the occurrence and development of asthma (10-12). Feng et al (13) demonstrated that the expression of miR-146a/b is upregulated in mouse spleen $\mathrm{CD}^{+} \mathrm{T}$ cells, and is positively correlated with the number of inflammatory cells in bronchoalveolar lavage fluid; following treatment with dexamethasone, the expression of miR-146a is significantly downregulated, indicating that $\mathrm{miR}-146 \mathrm{a} / \mathrm{b}$ may participate in the process of airway inflammation in asthma. Williams et al (14) demonstrated that the expression of miR-146a is elevated in airway biopsies of patients with mild asthma. In addition, the expression levels of miR-146a are increased in airway smooth muscles following stimulation by inflammatory factors (15). These studies suggest that elevated 
miR-146a levels may be associated with BSMC proliferation and apoptosis. However, to date, changes in miR-146a expression levels in the serum of children with asthma have yet to be reported. The present study aimed to determine changes in the serum expression levels of miR-146a in children with asthma, and to investigate the effect of miR-146a on BSMCs.

\section{Materials and methods}

Patients. A total of 60 children, including 30 with asthma and 30 healthy controls, were enrolled in the present study at the Maternal and Child Healthcare Hospital (Laiwu, China), General Hospital of Yanzhou Mining Bureau (Jining, China), Zoucheng People's Hospital (Zoucheng, China) and Dezhou People's Hospital (Dezhou, China) between January 2014 and December 2014. The 30 children with asthma included 13 girls with an average age of $10.46 \pm 4.29$ years and 17 boys with an average age of $10.86 \pm 3.56$ years. The exclusion criteria were as follows: i) Oral intake or intravenous injection of glucocorticoids or immunomodulators in the previous 2 weeks; ii) first-time asthma; iii) the presence of other immunologic diseases; and iv) cardiopulmonary failure or other malignant diseases. The 30 children in the control group included 13 girls with an average age of $10.89 \pm 3.15$ years and 17 boys with an average age of $11.23 \pm 2.90$ years. Children were enrolled in the control group if they lacked a history of asthma, recent respiratory tract infections or other malignant diseases. All procedures were approved by the Ethics Committee of the Taishan Medical College (Taian, China). Written-informed consent was obtained from the guardians of all patients.

Cell line and cell culture. Human BSMCs were purchased from Sciencell Research Laboratories (Carlsbad, CA, USA) and $5 \times 10^{4} \mathrm{BSMCs} / \mathrm{cm}^{2}$ were cultured in smooth muscle culture medium (Sciencell Research Laboratories, Carlsbad, CA, USA), supplemented with $5 \%$ fetal bovine serum (Invitrogen; Thermo Fisher Scientific, Inc., Waltham, MA, USA), $5 \mu \mathrm{g} / \mathrm{ml}$ insulin, $2 \mu \mathrm{g} / \mathrm{ml}$ human fibroblast growth factor, $50 \mathrm{ng} / \mathrm{ml}$ gentamicin, and $50 \mathrm{ng} / \mathrm{ml}$ amphotericin B (all Sigma-Aldrich, St. Louis, MO, USA) at $37^{\circ} \mathrm{C}$ in an atmosphere containing $5 \% \mathrm{CO}_{2}$.

miR-146a transfection. For transfection with miR-146a, BSMCs $\left(2 \times 10^{3} / \mathrm{cm}^{2}\right)$ were first seeded onto culture plates. When the cells reached 50-70\% confluency, they were transfected with $50 \mathrm{nM}$ miR-146a mimics, $100 \mathrm{nM}$ miR-146a inhibitor, or $50 \mathrm{nM}$ negative control using riboFECT CP (all Guangzhou RiboBio Co., Ltd., Guangzhou, China), according to the manufacturer's protocol. The cells were then cultured at $37^{\circ} \mathrm{C}$ in an atmosphere containing $5 \% \mathrm{CO}_{2}$.

Cell counting kit-8 (CCK-8) assay. For the CCK-8 assay (Beyotime Institute of Biotechnology, Haimen, China), cells $\left(5 \times 10^{3} / \mathrm{cm}^{2}\right)$ were seeded onto 96 -well plates in triplicate. A total of $24 \mathrm{~h}$ after inoculation, the cells were transfected as described above. At $6 \mathrm{~h}$ post-transfection, the transfection medium was replaced with fresh medium. At $0,12,24$, and $48 \mathrm{~h}$, WST reagent $(10 \mu \mathrm{l})$ was added to the cells. After $1 \mathrm{~h}$ culture at $37^{\circ} \mathrm{C}$ and $5 \% \mathrm{CO}_{2}$, the absorbance was measured at $450 \mathrm{~nm}$. For the determination of caspase-3/7 activity, the cells were also seeded onto 96 -well plates in triplicate, and transfected as described above. At 48 h post-transfection, caspase-3/7 activity was determined using a Caspase-Glo 3/7 kit (cat. no. G8090; Promega Corporation, Madison, WI, USA) according to the manufacturer's protocol.

Reverse transcription-quantitative polymerase chain reaction $(R T-q P C R)$. Total RNA $(1 \mu \mathrm{g})$ was extracted from the plasma $(200 \mu \mathrm{l})$ using an miRNeasy Serum/Plasma kit (cat. no. 217184; Qiagen GmbH, Hilden, Germany) following the manufacturer's protocol. Reverse transcription (5 $\mu \mathrm{l}$ RNA) was performed using a miScript II RT kit (cat. no. 218160; Qiagen $\mathrm{GmbH}$ ). Following dilution of the cDNA (10 times) in RNase-free water, $2 \mu \mathrm{l}$ cDNA was used for qPCR using the StepOnePlus ${ }^{\mathrm{TM}}$ Real-Time PCR System (Invitrogen; Thermo Fisher Scientific, Inc.), according to the protocol provided by the manufacturer of the miScript SYBR Green PCR kit (Qiagen $\mathrm{GmbH}$ ). Each sample was analyzed in triplicate and negative (no cDNA template) and reverse transcriptase-minus controls were performed. The $\mathrm{Cq}$ value of the plasma miR-146a was corrected using cel-miR-39 as an external reference, and the relative quantification of the target genes was determined using the $2^{-\Delta \Delta \mathrm{Cq}}$ method (16). The primers for miR-146a and cel-miR-39 were purchased from Qiagen. The PCR amplification protocol was as follows: Initial denaturation at $94^{\circ} \mathrm{C}$ for $15 \mathrm{~min} ; 40$ cycles of denaturation at $94^{\circ} \mathrm{C}$ for $10 \mathrm{sec}$, annealing at $55^{\circ} \mathrm{C}$ for $30 \mathrm{sec}$, and elongation at $70^{\circ} \mathrm{C}$ for $30 \mathrm{sec}$.

Western blotting. The cells were lysed using radioimmunoprecipitation assay lysis buffer (Beyotime Institute of Biotechnology), followed by centrifugation at $12,000 \mathrm{x} \mathrm{g}$ for 5 min at $4{ }^{\circ} \mathrm{C}$. The protein concentration in the supernatant was determined by performing a bicinchoninic acid assay. A total of $30 \mu \mathrm{g}$ protein was separated using $8 \%$ sodium dodecyl sulfate-polyacrylamide gel electrophoresis and transferred onto a nitrocellulose membrane. The membrane was blocked with $5 \%$ skimmed milk at room temperature for $1 \mathrm{~h}$, and incubated overnight at $4^{\circ} \mathrm{C}$ with mouse anti-phosphorylated (p)-signal transducer and activator of transcription 3 (stat3) monoclonal antibody (1:1,000; cat. no. 4113; Cell Signaling Technology, Inc., Danvers, MA, USA), rabbit anti-B-cell lymphoma 2 (Bcl-2) monoclonal antibody (1:1,000; cat. no. 2870; Cell Signaling Technology, Inc.), rabbit anti-p-extracellular regulated protein kinase (ERK) polyclonal antibody (1:800; cat. no. ab47339; Abcam, Cambridge, UK), rabbit anti-p-epidermal growth factor receptor (EGFR) monoclonal antibody (1:1,000; cat. no. 3777; Cell Signaling Technology, Inc.), rabbit anti-EGFR monoclonal antibody (1:1,000; cat. no. 2085; Cell Signaling Technology, Inc.) and rabbit anti-glyceraldehyde 3-phosphate dehydrogenase (GAPDH) monoclonal antibody (1:5,000; cat. no. 2118; Cell Signaling Technology, Inc.). Following 3 washes with Tris-buffered saline with Tween-20 (TBST; Sigma-Aldrich), the membrane was incubated with the secondary antibody (1:5,000 dilution) at room temperature for $1 \mathrm{~h}$. Following a further wash with TBST, electrochemiluminescent liquid was added. The images were of the membrane were subsequently captured using a Fusion Solo 4 Chemiluminescence system (Fisher Biotec Pty, Ltd., Wembley, WA, Australia), and analyzed using Quantity One 


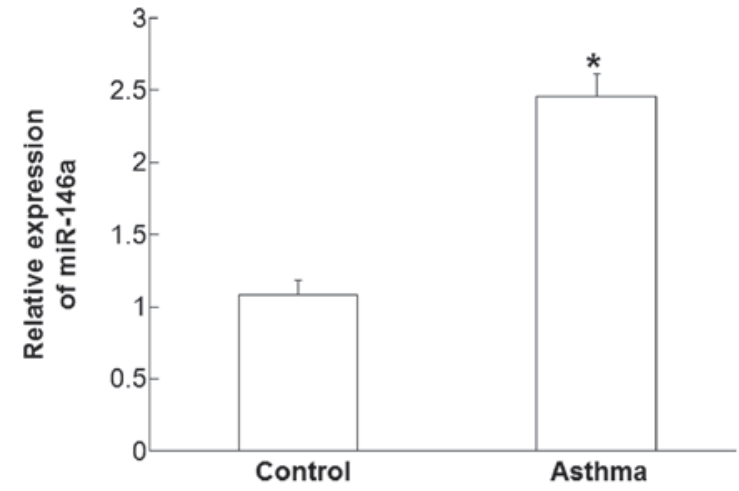

Figure 1. Relative expression levels of miR-146a in the plasma of healthy children and children with asthma, as determined by reverse transcription-quantitative polymerase chain reaction. The expression levels of miR-146a were upregulated in the children with asthma, as compared with the healthy children. Data are expressed as the mean \pm standard deviation. * $\mathrm{P}<0.05$, vs. the control. miR, microRNA.

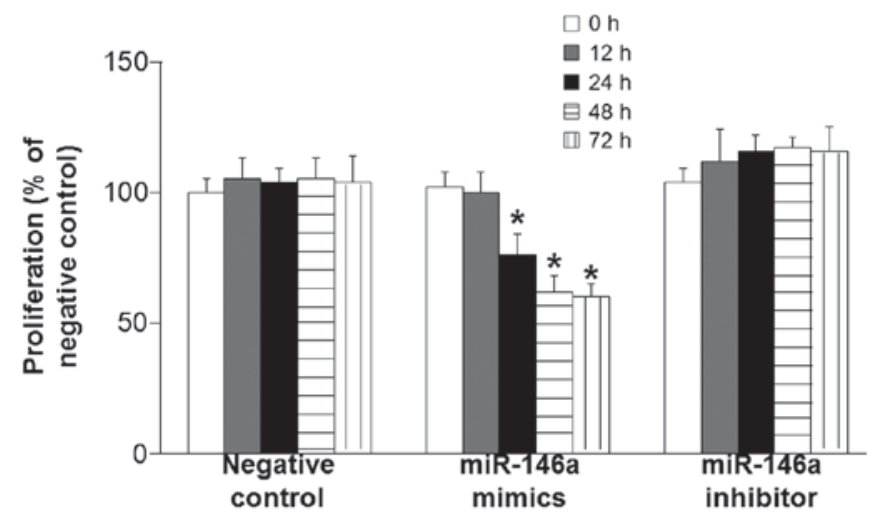

Figure 2. Proliferation of bronchial smooth muscle cells (BSMCs). The proliferation of BSMCs was decreased at 24,48 and $72 \mathrm{~h}$ following transfection with miR-146a mimics. Conversely, transfection with miR-146a inhibitor had no significant effect on the proliferation of BSMCs. Data are expressed as the mean \pm standard deviation. ${ }^{*} \mathrm{P}<0.05$, vs. the value at $0 \mathrm{~h}$ in the same group. miR, microRNA.

software, version 4.4.0 (Bio-Rad Laboratories, Inc., Hercules, CA, USA).

Bioinformatics analysis. Bioinformatics is a powerful tool for investigating the functions of miRNAs. In order to elucidate the regulatory roles of miR-146a, TargetScan 5.0 (http://www. targetscan.org) was used to predict the target molecules of miR-146a.

Dual-luciferase reporter assay. The wild-type 3'-untranslated region (UTR) of the EGFR mRNA that binds miR-146a and relevant mutant 3'-UTR were cloned into a psiCHECK reporter vector (Promega Corporation) to obtain wt-EGFR-3'-UTR vector and mut-EGFR-3'-UTR vector. The wt-EGFR-3'-UTR vector or mut-EGFR-3'-UTR vector was transfected into 293T cells (American Type Culture Collection, Manassas, VA, USA) together with control plasmids, and miR-146a or negative control mimics (Gene Copoeia, Inc., Rockville, MD, USA). The cells were lysed using lysis buffer (Promega Corporation) and the fluorescence intensity was determined using a GloMax 20/20

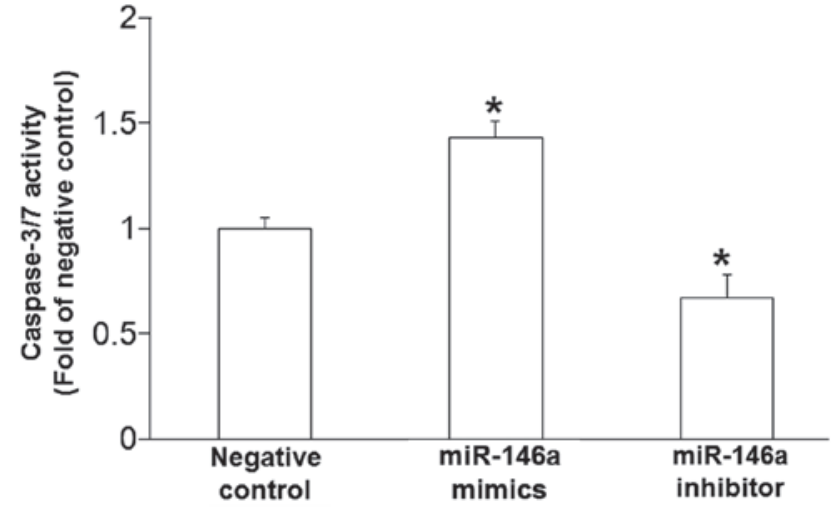

Figure 3. Activity of caspase-3/7 in bronchial smooth muscle cells (BSMCs). Caspase-3/7 activity was significantly increased in BSMCs transfected with miR-146a mimics, as compared with the negative control. Conversely, caspase-3/7 activity was significantly decreased in BSMCs transfected with miR-146a inhibitor, as compared with the negative control. Data are expressed as the mean \pm standard deviation. ${ }^{*} \mathrm{P}<0.05$, vs. the negative control. miR, microRNA.

luminometer (Promega Corporation). Subsequently, a dual-luciferase reporter assay was performed using a Dual-Luciferase Reporter Assay system (Promega Corporation), according to the manufacturer's protocol. Renilla fluorescence served as an internal reference. All tests were performed in triplicate.

Statistical analysis. All analyses were performed using SPSS v19.0 software (IBM SPSS, Armonk, NY, USA). The data were presented as means \pm standard deviation $(n \geq 3)$. Differences between groups of data were compared using $t$-tests. $\mathrm{P}<0.05$ was considered to indicate a statistically significant result.

\section{Results}

Plasma levels of miR-146a are increased in children affected by asthma. RT-qPCR was used measure the plasma levels of miR-146a. The data demonstrated that the expression levels of miR-146a in the plasma of patients with asthma was significantly higher compared with those in the control group $(\mathrm{P}<0.05$; Fig. 1). These results suggest that the plasma levels of miR-146a are increased in children with asthma.

Enhanced miR-146a expression inhibits the proliferation of BSMCs. To determine the expression levels of miR-146a in BSMCs and its effect on cell proliferation, RT-qPCR and a CCK-8 assay were performed. RT-qPCR data revealed that miR-146a expression levels in BSMCs transfected with miR-146a mimics were 968.76 times those of the control group. In addition, miR-146a expression levels in BSMCs transfected with miR-146a inhibitor were $23.33 \%$ those of the control group, whereas the expression levels of miR-146a in BSMCs transfected with negative control were not significantly different from those of the control group. Moreover, the CCK-8 assay demonstrated that the proliferation of BSMCs transfected with mimics was inhibited after 24, 48, and $72 \mathrm{~h}(\mathrm{P}<0.05)$. However, transfection with miR-146a inhibitor had an insignificant effect on BSMC proliferation ( $\mathrm{P}>0.05$; Fig. 2). These results suggest that enhanced miR-146a expression inhibits the proliferation of BSMCs. 


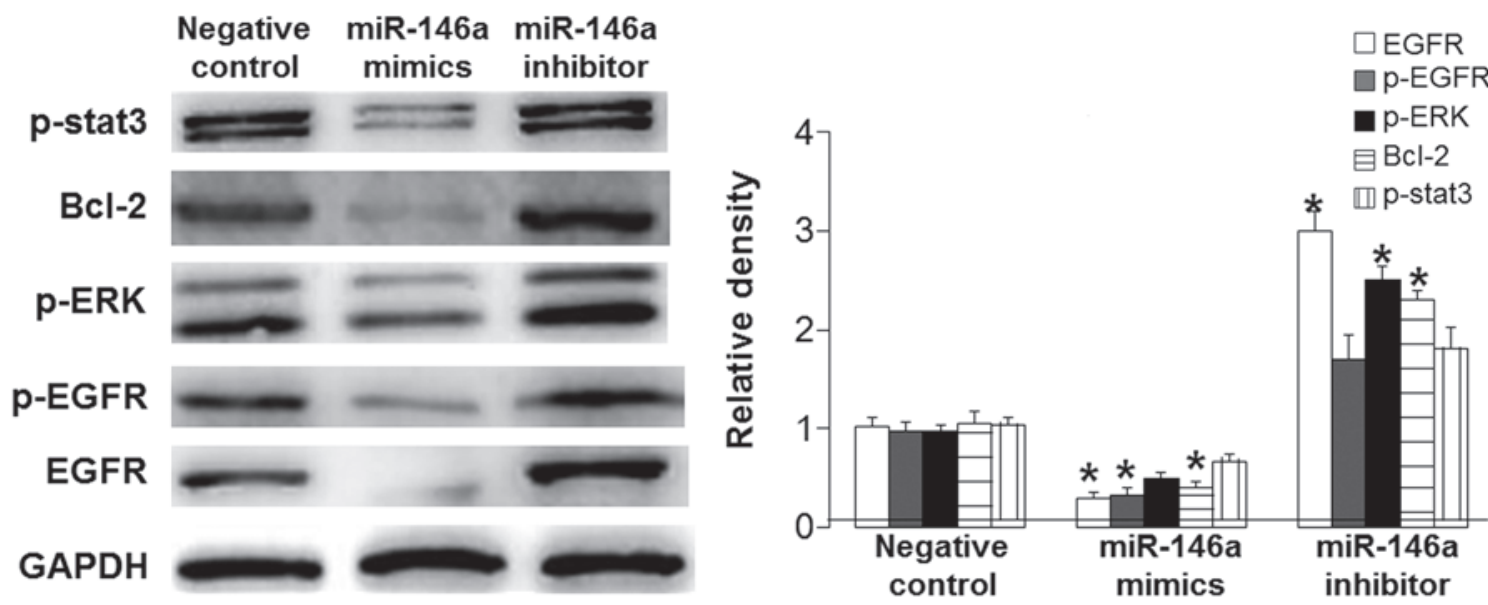

Figure 4. Effect of miR-146a on the protein expression levels of EGFR, p-EGFR, ERK, Bcl-2 and p-stat3. Western blotting was performed to determine the expression of the proteins. All data were expressed as the mean \pm standard deviation. "P<0.05, vs. the respective negative control. miR, microRNA; EGFR, epidermal growth factor receptor; ERK, extracellular regulated protein kinases; Bcl-2, B-cell lymphoma-2; p-stat3, phosphorylated signal transducers and activators of transcription 3 .

A

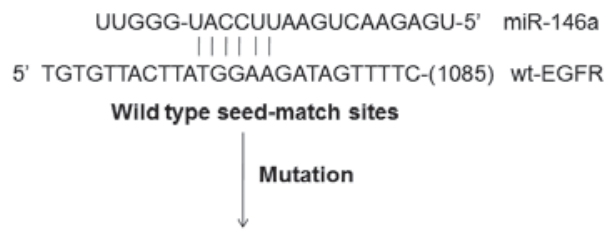

UUGGG-UACCUUAAGUCAAGAGU-5' miR-146a

5' TGTGTTACTTCACCTTGATAGTTTTC-(1085) mut-EGFR

Mutant seed-match sites

B
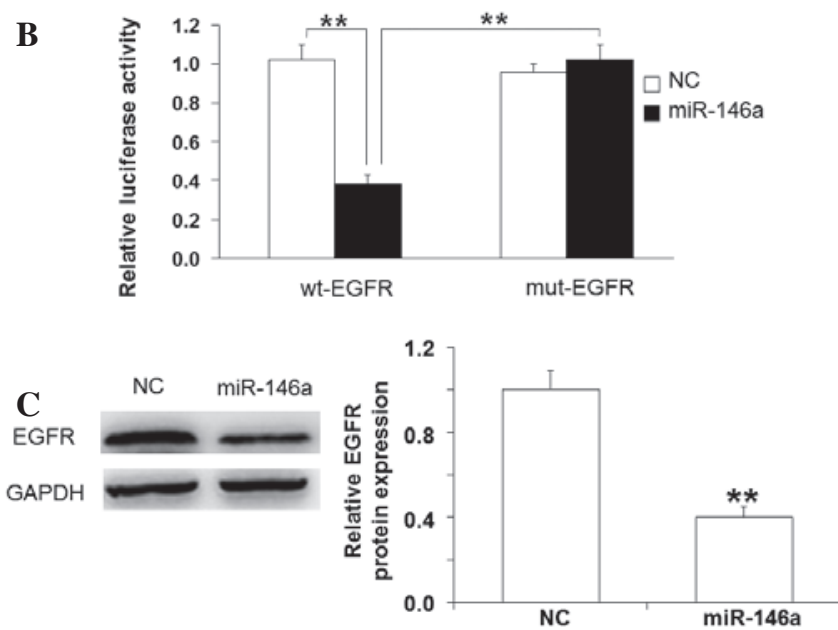

Figure 5. Targeting of miR-146a on EGFR.(A) Bioinformatics prediction of the interaction between miR-146a and the 3'-UTR of EGFR mRNA. (B) Relative luciferase activity of wt-EGFR-3'-UTR vector or mut-EGFR-3'-UTR vector in the negative control and miR-146a groups. ${ }^{* *} \mathrm{P}<0.01$, vs. the wt-EGFR group. (C) Protein expression of EGFR and miR146 as determined by western blotting and densitometry. ${ }^{* *} \mathrm{P}<0.01$ vs. the NC group. NC, negative control mimics; miR, microRNA; EGFR, epidermal growth factor receptor; wt, wild-type; mut, mutant.

BSMC apoptosis is promoted by miR-146a. To determine the effect of miR-146a on BSMC apoptosis, caspase-3/7 activity in BSMCs was examined $48 \mathrm{~h}$ post-transfection with miR-146a mimics and miR-146a inhibitor. The data demonstrated that miR-146a mimics significantly enhanced the activity of caspase-3/7 in BSMCs, whereas miR-146a inhibitor significantly decreased the activity of caspase-3/7 $(\mathrm{P}<0.05$; Fig. 3). These results suggest that miR-146a promotes BSMC apoptosis.

$T$ mechanism underlying miR-146a-induced BSMC apoptosis is its direct targeting of EGFR that affects downstream signaling pathways. To determine the signaling pathway by which miR-146a promotes BSMC apoptosis, western blotting, bioinformatics and a dual-luciferase reporter assay were performed. Western blotting data showed that miR-146a mimics significantly reduced the protein expression levels of EGFR and p-EGFR $(\mathrm{P}<0.05)$, markedly downregulated the ERK signaling pathway and p-stat3 expression levels, and significantly decreased $\mathrm{Bcl}-2$ expression levels $(\mathrm{P}<0.05)$. However, these effects were reversed by miR-146a inhibitor (Fig. 4). Using bioinformatics tools, it was demonstrated that miR-146a may bind to the 3'-UTR of EGFR mRNA (Fig. 5A). Dual-luciferase reporter assay demonstrated that miR-146a mimics significantly reduced luciferase activity of wt-EGFR compared with negative control, but had no significant effect on that of mut-EGFR (Fig. 5B). Western blotting demonstrated that EGFR was targeted by miR-146a (Fig. 5C). These results suggest that the mechanism underlying miR-146a-induced promotion of BSMC apoptosis is its direct targeting of EGFR that affects downstream signaling pathways.

\section{Discussion}

The present study first investigated the association between plasma miR-146a expression levels and pediatric asthma. The results demonstrated that children with asthma had significantly higher miR-146a expression levels compared with normal subjects, indicating that miR-146a may have a role in pediatric asthma. miR-146a is an important regulator of immunoreaction and inflammatory diseases (17). A previous study reported that the activity of $\mathrm{T}$ cells with knockout of miR-146a was increased in acute and chronic inflammatory processes (3). However, another study reported that the 
expression of miR-146a was upregulated in spleen CD4 ${ }^{+}$ $\mathrm{T}$ cells in the ovalbumin-induced mouse asthma model (2). Liu et al (18) demonstrated that miR-146a facilitates the survival of human bronchial epithelial cells by upregulating the phosphorylation levels of B-cell lymphoma-extra large and signal transducers and activators of transcription 3 , leading to the restoration and remodeling of tissues (18). Elevated miR-146a expression in airway smooth muscle cells that is stimulated by inflammatory factors negatively regulates the levels of interleukin- $\beta$ (15). The results of the present study demonstrated that miR-146a inhibited the proliferation and promoted the apoptosis of BSMCs.

EGFR is a type of receptor tyrosine kinase that is widely distributed in human cell membranes (19). The immunoreactivity of EGFR is enhanced in the bronchial smooth muscles of patients with asthma, and inhibitors of EGFR are able to reduce collagen deposition and mucus accumulation following antigen processing, suggesting that EGFR may participate in airway remodeling (20-23). Xu et al (24) demonstrated that miR-146a inhibits the proliferation of prostate cancer cells by regulating the EGFR signaling pathway. Park et al (25) reported that miR-146a affects the apoptosis of dendritic cells by targeting tumor necrosis factor receptor-associated factor 6 and interleukin-1 receptor-associated kinase 1 . The present study demonstrated that miR-146a downregulated the expression of EGFR and p-EGFR, and reduced the levels of p-ERK and p-stat3, suggesting that miR-146a altered the proliferation and apoptosis of BSMCs via the p-ERK-dependent signaling pathway. In addition, miR-146a increased caspase 3/7 activity and reduced $\mathrm{Bcl}-2$ expression, indicating that caspases participate in BSMC apoptosis.

In conclusion, the present study demonstrated that an increase in miR-146a expression in patients with asthma inhibits the proliferation and promotes the apoptosis of BSMCs. The study provides a novel clinical marker for the diagnosis and treatment of asthma, and an experimental basis for its molecular targeted therapy.

\section{Acknowledgements}

The authors of the present study would like to acknowledge Professor Xuechun Wang of Taishan Medical College for his support and comments.

\section{References}

1. Lochte L, Nielsen KG, Petersen PE and Platts-Mills TA: Childhood asthma and physical activity: A systematic review with meta-analysis and Graphic Appraisal Tool for Epidemiology assessment. BMC Pediatr 16: 50, 2016.

2. Yang L, Boldin MP, Yu Y, Liu CS, Ea CK, Ramakrishnan P, Taganov KD, Zhao JL and Baltimore D: miR-146a controls the resolution of T cell responses in mice. J Exp Med 209: 1655-1670, 2012.

3. Omran A, Elimam D and Yin F: MicroRNAs: New insights into chronic childhood diseases. BioMed Res Int 2013: 291826 , 2013.

4. Tsitsiou E, Williams AE, Moschos SA, Patel K, Rossios C, Jiang X, Adams OD, Macedo P, Booton R, Gibeon D, et al: Transcriptome analysis shows activation of circulating CD8+ T cells in patients with severe asthma. J Allergy Clin Immunol 129: 95-103, 2012.

5. Dileepan M, Sarver AE, Rao SP, Panettieri RA Jr, Subramanian S and Kannan MS: MicroRNA Mediated Chemokine Responses in Human Airway Smooth Muscle Cells. PLoS One 11: e0150842, 2016
6. Bara I, Ozier A, Tunon de Lara JM, Marthan R and Berger P: Pathophysiology of bronchial smooth muscle remodelling in asthma. Eur Respir J 36: 1174-1184, 2010.

7. Roviezzo F, Sorrentino R, Bertolino A, De Gruttola L, Terlizzi M, Pinto A, Napolitano M, Castello G, D'Agostino B, Ianaro A, et al: S1P-induced airway smooth muscle hyperresponsiveness and lung inflammation in vivo: Molecular and cellular mechanisms. Br J Pharmacol 172: 1882-1893, 2015.

8. Perry MM, Baker JE, Gibeon DS, Adcock IM and Chung KF: Airway smooth muscle hyperproliferation is regulated by microRNA-221 in severe asthma. Am J Respir Cell Mol Biol 50:ح7-17, 2014.

9. Rebane A: microRNA and Allergy. Adv Exp Med Biol 888: 331-352, 2015.

10. Kuhn AR, Schlauch K, Lao R, Halayko AJ, Gerthoffer WT and Singer CA: MicroRNA expression in human airway smooth muscle cells: Role of miR-25 in regulation of airway smooth muscle phenotype. Am J Respir Cell Mol Biol 42: 506-513, 2010.

11. Hu R, Pan W, Fedulov AV, Jester W, Jones MR, Weiss ST, Panettieri RA Jr, Tantisira K and Lu Q: MicroRNA-10a controls airway smooth muscle cell proliferation via direct targeting of the PI3 kinase pathway. FASEB J 28: 2347-2357, 2014.

12. Pua HH and Ansel KM: MicroRNA regulation of allergic inflammation and asthma. Curr Opin Immunol 36: 101-108, 2015.

13. Feng MJ, Shi F, Qiu C and Peng WK: MicroRNA-181a, -146a and $-146 \mathrm{~b}$ in spleen CD4+ T lymphocytes play proinflammatory roles in a murine model of asthma. Int Immunopharmacol 13: 347-353, 2012.

14. Williams AE, Larner-Svensson H, Perry MM, Campbell GA, Herrick SE, Adcock IM, Erjefalt JS, Chung KF and Lindsay MA: MicroRNA expression profiling in mild asthmatic human airways and effect of corticosteroid therapy. PloS One 4: e5889, 2009.

15. Comer BS, Camoretti-Mercado B, Kogut PC, Halayko AJ, Solway J and Gerthoffer WT: MicroRNA-146a and microRNA-146b expression and anti-inflammatory function in human airway smooth muscle. Am J Physiol Lung Cell Mol Physiol 307: L727-L734, 2014.

16. Chen Y, Gelfond JA, McManus LM and Shireman PK: Reproducibility of quantitative RT-PCR array in miRNA expression profiling and comparison with microarray analysis. BMC Genomics 10: 407, 2009.

17. Saba R, Sorensen DL and Booth SA. MicroRNA-146a: A Dominant, Negative Regulator of the Innate Immune Response. Front Immunol 5: 578, 2014.

18. Liu X, Nelson A, Wang X, Kanaji N, Kim M, Sato T, Nakanishi M, Li Y, Sun J, Michalski J, et al: MicroRNA-146a modulates human bronchial epithelial cell survival in response to the cytokine-induced apoptosis. Biochem Biophys Res Commun 380: 177-182, 2009.

19. Tan X, Lambert PF, Rapraeger AC and Anderson RA: Stress-Induced EGFR Trafficking: Mechanisms, Functions, and Therapeutic Implications. Trends Cell Biol 25: 352-366, 2016.

20. Wu W, Wages PA, Devlin RB, Diaz-Sanchez D, Peden DB and Samet JM: SRC-mediated EGF receptor activation regulates ozone-induced interleukin 8 expression in human bronchial epithelial cells. Environ Health Perspect 123: 231-236, 2015.

21. Hao Y, Kuang Z, Jing J, Miao J, Mei LY, Lee RJ, Kim S, Choe S, Krause DC and Lau GW: Mycoplasma pneumoniae modulates STAT3-STAT6/EGFR-FOXA2 signaling to induce overexpression of airway mucins. Infect Immun 82: 5246-5255, 2014.

22. Le Cras TD, Acciani TH, Mushaben EM, Kramer EL, Pastura PA, Hardie WD, Korfhagen TR, Sivaprasad U, Ericksen M, Gibson AM, et al: Epithelial EGF receptor signaling mediates airway hyperreactivity and remodeling in a mouse model of chronic asthma. Am J Physiol Lung Cell Mol Physiol 300: L414-L421, 2011.

23. Yoshikawa $\mathrm{T}$ and Kanazawa $\mathrm{H}$ : Integrated effect of EGFR and PAR-1 signaling crosstalk on airway hyperresponsiveness. Int J Mol Med 30: 41-48, 2012.

24. Xu B, Wang N, Wang X, Tong N, Shao N, Tao J, Li P, Niu X, Feng N, Zhang L, et al: MiR-146a suppresses tumor growth and progression by targeting EGFR pathway and in a p-ERK-dependent manner in castration-resistant prostate cancer. Prostate 72: 1171-1178, 2012.

25. Park H, Huang X, Lu C, Cairo MS and Zhou X: MicroRNA-146a and microRNA-146b regulate human dendritic cell apoptosis and cytokine production by targeting TRAF6 and IRAK1 proteins. J Biol Chem 290: 2831-2841, 2015 . 